\title{
ON CERTAIN SUMS OF FOURIER-STIELTJES COEFFICIENTS \\ BY
}

J. B. TWOMEY

\begin{abstract}
We obtain estimates for certain sums of Fourier-Stieltjes (and hence also Fourier) coefficients of continuous functions $f$ of bounded variation in terms of the modulus of continuity of $f$. As a consequence of one of our results we obtain an improvement on a theorem of Zygmund on the absolute convergence of Fourier series of functions of bounded variation. We also consider absolutely continuous functions and show by examples that a number of the results we obtain are "best possible".
\end{abstract}

1. Introduction. We shall be concerned with $\mathrm{BV}$, the class of (complex-valued) functions $f$ which have bounded variation on $[0,2 \pi]$, are continuous there and are defined for all $x$ by the condition

$$
f(x+2 \pi)-f(x)=f(2 \pi)-f(0), \quad-\infty<x<\infty,
$$

and also with AC, the subclass of functions in BV that are absolutely continuous. Throughout the paper we denote by $c_{n}=c_{n}(f)$ the $n$th Fourier-Stieltjes coefficient of a function $f \in \mathrm{BV}$, that is,

$$
c_{n}=\frac{1}{2 \pi} \int_{0}^{2 \pi} e^{-i n t} d f(t), \quad n=0, \pm 1, \pm 2, \ldots
$$

For $\delta>0$ we also write

$$
\omega(\delta)=\omega(\delta, f)=\sup \left\{\left|f\left(x_{1}\right)-f\left(x_{2}\right)\right|:\left|x_{1}-x_{2}\right| \leqslant \delta\right\},
$$

so that $\omega$ is the modulus of continuity function of $f$.

We have the following classical result of Wiener [1, Vol. 1, p. 212; 8]:

Let $f \in \mathrm{BV}$. Then

$$
\sum_{k=-n}^{n}\left|c_{k}\right|^{2} \leqslant K V_{f} n \omega\left(n^{-1}\right), \quad n \geqslant 1
$$

Here and throughout $K, K_{1}$ and $K_{2}$ denote absolute positive constants, not usually the same at each occurrence, and $V_{f}$ denotes the total variation of $f$ over $[0,2 \pi]$. In this paper we derive an inequality which is stronger than Wiener's result, obtain a refinement of (1.1) for functions in $\mathrm{AC}$, and derive a number of inequalities similar to (1.1) for certain other sums of Fourier-Stieltjes coefficients. As a corollary to one of our results, we obtain an improvement of a theorem of Zygmund on the absolute

Received by the editors September 22, 1982.

1980 Mathematics Subject Classification. Primary 42A16, Secondary 42A28.

Key words and phrases. Fourier-Stieltjes coefficients, coefficient estimates, functions of bounded variation, absolutely continuous functions, modulus of continuity, absolutely convergent Fourier serics. 
convergence of Fourier series of functions of bounded variation. We also show by examples that a number of the results we obtain are "best possible".

2. Some definitions and lemmas. A nonnegative function $\psi$, defined on $(0, \infty)$, is said to be a function of continuity modulus type, and we write $\psi \in \mathrm{CMT}$, if

(i) $\psi$ is increasing on $(0, \infty)$,

(ii) $\psi(\delta) \rightarrow 0\left(\delta \rightarrow 0^{+}\right)$, and

(iii) $\psi(n \delta) \leqslant n \psi(\delta)$ for every $\delta>0$ and every positive integer $n$.

Lemma 1. Let $\psi \in \mathrm{CMT}$. Then, if $0<s \leqslant t$,

$$
t^{-1} \psi(t) \leqslant 2 s^{-1} \psi(s)
$$

Proof. Let $k$ be the positive integer for which $k s \leqslant t<(k+1) s$. Then, by (i) and (iii) above,

$$
t^{-1} \psi(t) \leqslant(k s)^{-1} \psi((k+1) s) \leqslant(k s)^{-1}(k+1) \psi(s) \leqslant 2 s^{-1} \psi(s) .
$$

Since, trivially, $\omega \in \mathrm{CMT}$ for the modulus of continuity function $\omega$ of a function $f \in \mathrm{BV}$, it thus follows that, for such an $\omega$,

$$
t^{-1} \omega(t) \leqslant 2 s^{-1} \omega(s), \quad 0<s \leqslant t .
$$

We define the convolution function $f^{*}$ for $f \in \mathrm{BV}$ by

$$
f^{*}(x)=\frac{1}{2 \pi} \int_{0}^{2 \pi} f(x+t) d \overline{f(t)}, \quad-\infty<x<\infty .
$$

Clearly $f^{*} \in \mathrm{BV}$. For $f \in \mathrm{BV}$ we also write

$$
\mu(\delta, f)=\sup \{|f(x)-f(0)|: 0 \leqslant x \leqslant \delta\} .
$$

Lemma 2. Let $f \in \mathrm{AC}$ and suppose $\delta^{-1} \omega(\delta, f) \rightarrow \infty\left(\delta \rightarrow 0^{+}\right)$. Then

$$
\mu\left(\delta, f^{*}\right)=o(\omega(\delta, f)), \quad \delta \rightarrow 0^{+} .
$$

Proof. Let

$$
f_{h}(t)=\frac{f(t+h)-f(t)}{h}, \quad F_{h}(t)=\frac{h f_{h}(t)}{\omega(|h|, f)},
$$

for $t$ in $[0,2 \pi]$ and $h \neq 0$. Clearly

$$
\left|F_{h}(t)\right| \leqslant 1
$$

for $t$ in $[0,2 \pi]$ and $h \neq 0$. Since $f \in A C$, there exists $g \in L(0,2 \pi)$ such that $f_{h}(t) \rightarrow g(t)$ a.e. as $h \rightarrow 0$, and it follows, since $h^{-1} \omega(|h|, f) \rightarrow \infty(h \rightarrow 0)$, that

$$
F_{h}(t) \rightarrow 0 \text { a.e. as } h \rightarrow 0 \text {. }
$$

Now

$$
2 \pi f^{*}(x)=\int_{0}^{2 \pi} f(x+t) d \overline{f(t)}=\int_{0}^{2 \pi} f(x+t) \overline{g(t)} d t
$$

so, for $h>0$,

$$
2 \pi \frac{\left[f^{*}(h)-f^{*}(0)\right]}{\omega(h, f)}=\int_{0}^{2 \pi} F_{h}(t) \overline{g(t)} d t
$$


and, by (2.4), (2.5) and Lebesgue's dominated convergence theorem, this last integral tends to 0 as $h \rightarrow 0^{+}$. The required result follows immediately from this and the monotonicity of $\omega$.

For $n \geqslant 1$ and $f \in \mathrm{BV}$, we set

$$
\sigma_{n}(x)=\sigma_{n}(x, f)=\left(n^{-1}\right)\left(s_{0}+s_{1}+\cdots+s_{n-1}\right)
$$

where

$$
s_{n}=s_{n}(x, f)=\sum_{k=-n}^{n} c_{k} e^{i k x}, \quad n=0,1,2, \ldots,
$$

so that $\sigma_{n}(x)$ is the $n$th Cesàro mean of the Fourier-Stieltjes series of $f$.

LEMMA 3. Let $f$ be an increasing function in BV. Then, for each $x$ in $[0,2 \pi]$ and $n \geqslant 1$,

$$
K_{1} n\left[f\left(x+n^{-1}\right)-f\left(x-n^{-1}\right)\right] \leqslant \sigma_{n}(x) \leqslant K_{2} n \omega\left(n^{-1}, f\right) .
$$

ProOF. For $n \geqslant 1$ set

$$
\begin{aligned}
F_{n}(t) & =\frac{\sin ^{2} \frac{1}{2} n t}{n \sin ^{2} \frac{1}{2} t}, & & t \neq 0(\bmod 2 \pi), \\
& =n, & & \text { otherwise. }
\end{aligned}
$$

Then $F_{n}$ is the Fejer kernel of degree $n$ and, by a familiar formula,

$$
2 \pi \sigma_{n}(x)=\int_{-\pi}^{\pi} F_{n}(x-t) d f(t)=\int_{0}^{\pi} F_{n}(t) d \phi_{x}(t)
$$

for $n \geqslant 1$, where

$$
\phi_{x}(t)=f(x+t)-f(x-t)
$$

for all $x$ and $t$. It is readily shown that, for $0 \leqslant t \leqslant n^{-1}, F_{n}(t) \geqslant K n$ and hence

$$
2 \pi \sigma_{n}(x) \geqslant \int_{0}^{1 / n} F_{n}(t) d \phi_{x}(t) \geqslant K n \phi_{x}\left(n^{-1}\right),
$$

where we have used the monotonicity of $f$. This gives the left-hand inequality of (2.6).

We note next (see [5, p. 62]) that if $\alpha$ is a suitably chosen constant, and

$$
G_{n}(t)=\alpha n /\left(1+n^{2} t^{2}\right),
$$

then $F_{n}(t) \leqslant G_{n}(t), 0 \leqslant t \leqslant \pi$, and

$$
\int_{0}^{\pi} t\left|G_{n}^{\prime}(t)\right| d t \leqslant K_{1}
$$

Hence

$$
2 \pi \sigma_{n}(x) \leqslant \int_{0}^{\pi} G_{n}(t) d \phi_{x}(t),
$$

and integrating by parts we obtain

$$
2 \pi \sigma_{n}(x) \leqslant G_{n}(\pi) \phi_{x}(\pi)-\int_{0}^{\pi} \phi_{x}(t) G_{n}^{\prime}(t) d t .
$$


Clearly,

$$
\left|G_{n}(\pi) \phi_{x}(\pi)\right| \leqslant \alpha n^{-1} \omega(2 \pi, f) \leqslant \alpha 4 \pi \omega\left(n^{-1}, f\right)
$$

by (2.2). Also, since $G_{n}^{\prime}(t) \leqslant 0$ for $t \geqslant 0$,

$$
\begin{aligned}
0 & \leqslant-\int_{0}^{\pi} \phi_{x}(t) G_{n}^{\prime}(t) d t \leqslant \int_{0}^{\pi} \omega(2 t, f)\left[-G_{n}^{\prime}(t)\right] d t \\
& \leqslant \omega\left(2 n^{-1}\right) \int_{0}^{1 / n}\left[-G_{n}^{\prime}(t)\right] d t+2 n \omega\left(2 n^{-1}\right) \int_{1 / n}^{\pi} t\left[-G_{n}^{\prime}(t)\right] d t \\
& \leqslant 2 \omega\left(n^{-1}\right) G_{n}(0)+4 n \omega\left(n^{-1}\right) \int_{0}^{\pi} t\left[-G_{n}^{\prime}(t)\right] d t,
\end{aligned}
$$

where we have again used (2.2). The right-hand inequality of (2.6) now follows from this and (2.7).

REMARK. It can be shown that the right-hand inequality of (2.6) holds for every $f \in \mathrm{BV}$. The result proved here is sufficient for our purposes, however.

We make two final definitions. We write $\mathrm{BV}^{+}\left(\mathrm{AC}^{+}\right)$to denote the subclass of functions in BV (AC) for which $c_{n}(f) \geqslant 0$ for every $n$ and, for $\psi \in \mathrm{CMT}$, we write $H[\psi]$ to denote the class of all functions $f \in \mathrm{BV}$ satisfying $\omega(\delta, f) \leqslant K \psi(\delta), \delta>0$.

3. Results. We begin with a result for the class $\mathrm{BV}^{+}$.

THEOREM 1. Let $f \in \mathrm{BV}^{+}$. Then, for $n \geqslant 1$,

$$
\sum_{k=-n}^{n} c_{k} \leqslant K n\left[\mu\left(n^{-1}, f\right)+c_{0} n^{-1}\right]
$$

Proof. Let $f \in \mathrm{BV}^{+}$and set

$$
F(x)=f(x)-c_{0} x, \quad x \in[0,2 \pi] .
$$

Then $F \in \mathrm{BV}$ and $F(2 \pi)=\mathrm{F}(0)$, since $2 \pi c_{0}=f(2 \pi)-f(0)$. Hence, for $k \neq 0$.

$$
\int_{0}^{2 \pi} e^{-i k x} F(x) d x=i k^{-1} \int_{0}^{2 \pi} e^{-i k x} d f(x)=2 \pi i k^{-1} c_{k},
$$

and, since $\operatorname{Re} F$ is a continuous periodic function of bounded variation, its Fourier series, namely

$$
\frac{1}{2} a_{0}-\sum_{k=1}^{\infty}\left(c_{k}+c_{-k}\right) k^{-1} \sin k x,
$$

converges to $\operatorname{Re} F(x)$ for all $x$. In particular, $a_{0}=2 \operatorname{Re} F(0)$. Since Fourier series can be integrated term-by-term, we have (cf. [2, p. 469])

$$
\begin{aligned}
\operatorname{Re} \int_{0}^{x}[F(0)-F(t)] d t & =\sum_{k=1}^{\infty} \alpha_{k} k^{-2}(1-\cos k x) \\
& =2 \sum_{k=1}^{\infty} \alpha_{k} k^{-2} \sin ^{2} \frac{1}{2} k x,
\end{aligned}
$$

where $\alpha_{k}=c_{k}+c_{-k}, k \geqslant 1$. Hence, for $x \geqslant 0$ and every integer $n \geqslant 1$,

$$
2 \sum_{k=1}^{n} \alpha_{k} k^{-2} \sin ^{2} \frac{1}{2} k x \leqslant \int_{0}^{x}|F(0)-F(t)| d t \leqslant x \mu(x, f)+c_{0} x^{2} \text {. }
$$


Taking $x=n^{-1}$ and using the inequalities $\sin (k / 2 n) \geqslant k / n \pi, 1 \leqslant k \leqslant n$, we obtain

$$
2 \sum_{k=1}^{n} \alpha_{k} \leqslant n \pi^{2}\left[\mu\left(n^{-1}, f\right)+c_{0} n^{-1}\right] .
$$

This proves the theorem.

If $f \in \mathrm{BV}$, then the convolution function $f^{*} \in \mathrm{BV}^{+}$since $c_{n}\left(f^{*}\right)=\left|c_{n}(f)\right|^{2}$, $n=0, \pm 1, \pm 2, \ldots[11$, Vol. 1, p. 40]. Our second theorem thus follows immediately.

THEOREM 2. Let $f \in \mathrm{BV}$ and let $f^{*}$ be defined by (2.3). Then

$$
\sum_{h=-n}^{\prime \prime}\left|c_{h}(f)\right|^{2} \leqslant K n\left[\mu\left(n^{-1}, f^{*}\right)+n^{-1}\left|c_{0}(f)\right|^{2}\right], \quad n \geqslant 1 .
$$

We note that (3.1) implies, for $f \in \mathrm{BV}^{+}$,

$$
\sum_{h=-n}^{n} c_{h}(f) \leqslant K n \omega\left(n^{-1}, f\right) \quad(n \geqslant 1)
$$

since $\mu\left(n^{-1}, f\right) \leqslant \omega\left(n^{-1}, f\right)$ and

$$
2 \pi c_{0}=f(2 \pi)-f(0) \leqslant \omega(2 \pi, f) \leqslant 4 \pi n \omega\left(n^{-1}, f\right),
$$

by (2.2). Similarly, since $2 \pi \omega\left(\delta, f^{*}\right) \leqslant V_{f} \omega(\delta, f), \delta>0,(3.2)$ implies Wiener's result (1.1).

Inequality (3.2) has an interesting corollary for Fourier series of functions of bounded variation. To derive this let $g$ be a continuous periodic function of period $2 \pi$ that has bounded variation on $[0,2 \pi]$. Then $g \in \mathrm{BV}$ and the complex Fourier coefficients $\left(\hat{g}_{k}\right)$ of $g$ satisfy $k \hat{g}_{k}=i c_{k}(g), k$ an integer. Hence, by (3.2) and the Cauchy-Schwarz inequality,

$$
\sum_{h}^{n} k\left|\hat{g}_{k}\right|=\sum_{k=1}^{n}\left|c_{k}(g)\right| \leqslant K n\left[\mu\left(n^{-1}, g^{*}\right)\right]^{1 / 2},
$$

and since, by partial summation,

$$
\sum_{h}^{n}\left|\hat{g}_{h}\right|=\sum_{h=1}^{n}[k(k+1)]^{-1}\left(\sum_{m=1}^{h} m\left|\hat{g}_{m}\right|\right)+(n+1)^{-1} \sum_{k=1}^{n} k\left|\hat{g}_{k}\right|
$$

for $n \geqslant 2$, we deduce immediately that $\sum_{k=1}^{\infty}\left|\hat{g}_{k}\right|<\infty$ if the series

$$
\sum_{k=1}^{\infty} k^{-1}\left[\mu\left(k^{-1}, g^{*}\right)\right]^{1 / 2}
$$

converges. Combining this with a similarly proved analogous result for the Fourier coefficients of negative index, we obtain

Corollary 1. If a function $g \in \mathrm{BV}$ is periodic with period $2 \pi$ and is such that the series (3.4) converges, then the Fourier series of $g$ is absolutely convergent.

The well-known theorem of Zygmund [11, Vol. 2, p. 160] that the absolute convergence of the Fourier series of a periodic function $g \in \mathrm{BV}$ is implied by the convergence of the series

$$
\sum_{k=1}^{\infty} k^{-1}\left[\omega\left(k^{-1}, g\right)\right]^{1 / 2}
$$


clearly follows from this corollary since convergence of (3.5) implies convergence of (3.4). We show that Corollary 1 is stronger than Zygmund's theorem by constructing, at the end of $\S 5$, a periodic function $g \in \mathrm{BV}$ for which (3.5) is divergent while (3.4) is convergent. In relation to Zygmund's result, we remark that the condition imposed on the modulus of continuity function has been shown to be best possible [3] (see also [9, 10]).

Our second corollary to Theorem 2 shows that Wiener's result (1.1) can be strengthened for the class AC.

Corollary 2. Let $f \in \mathrm{AC}$ and suppose $n \omega\left(n^{-1}, f\right) \rightarrow \infty(n \rightarrow \infty)$. Then

$$
\sum_{k=-n}^{n}\left|c_{k}\right|^{2}=o\left(n \omega\left(n^{-1}, f\right)\right), \quad n \rightarrow \infty .
$$

Equivalently, if $\phi$ is any integrable periodic function of period $2 \pi$ with complex Fourier coefficients $\left(\hat{\phi}_{k}\right)$, then

$$
\sum_{k=-n}^{n}\left|\hat{\phi}_{k}\right|^{2}=o\left(n \omega\left(n^{-1}, \Phi\right)\right), \quad n \rightarrow \infty,
$$

if $n \omega\left(n^{-1}, \Phi\right) \rightarrow \infty(n \rightarrow \infty)$, where $\Phi$ is an indefinite integral of $\phi$.

Proof. Estimate (3.6) is an immediate consequence of (3.2) and Lemma 2. The second part of the corollary then follows from the identities $c_{k}(\Phi)=\hat{\phi}_{k}, k$ any integer.

REMARK. (3.6), for the special case of increasing functions in AC, can also be deduced from results in [7].

The next result we state is readily deduced from (3.3) once it is noted that, for $f \in \mathrm{BV}, 2 \pi\left|c_{n}\right| \leqslant V_{f}, n$ any integer, while, for $f \in \mathrm{AC}, c_{n} \rightarrow 0(|n| \rightarrow \infty)$, since $\left(c_{n}\right)$ is then the sequence of Fourier coefficients of the integrable function $f^{\prime}$.

THEOREM 3. If $f \in \mathrm{BV}^{+}$then, for every $\lambda \geqslant 1$,

$$
\sum_{k=-n}^{n} c_{k}^{\lambda} \leqslant K V_{f}^{\lambda-1} n \omega\left(n^{-1}, f\right), \quad n \geqslant 1 .
$$

If $f \in \mathrm{AC}^{+}$and $n \omega\left(n^{-1}, f\right) \rightarrow \infty(n \rightarrow \infty)$, then

$$
\sum_{k=-n}^{n} c_{k}^{\lambda}=o\left(n \omega\left(n^{-1}, f\right)\right), \quad n \rightarrow \infty,
$$

for every $\lambda>1$.

Obviously, because of (3.1), we can replace $\omega\left(n^{-1}, f\right)$ by $\mu\left(n^{-1}, f\right)+c_{0} n^{-1}$ in (3.7) and by $\mu\left(n^{-1}, f\right)$ in (3.8) if $n \mu\left(n^{-1}, f\right) \rightarrow \infty(n \rightarrow \infty)$. However, as estimates in terms of the modulus of continuity function, each of the inequalities (3.7) and (3.8) is best possible within its class for every $\lambda>1$ however large. We prove this in $\S 4$ (Theorems 6,7) with the help of some examples of Salem. In $\$ 5$ we show that (3.7) 
with $\lambda=1$ is the best possible result of its kind even for the class $\mathrm{AC}^{+}$by proving

ThEOREM 4. Let $\psi \in \mathrm{CMT}$ be such that $n \psi\left(n^{-1}\right) \rightarrow \infty(n \rightarrow \infty)$. Then there exists $f \in \mathrm{AC}^{+} \cap H[\psi]$ for which

$$
\sum_{k=-n}^{n} c_{k}(f) \geqslant K n \psi\left(n^{-1}\right) \text { for every } n \geqslant 1 .
$$

It is clear, because of (1.1), (3.6), and the inequalities $2 \pi\left|c_{k}\right| \leqslant V_{f}(f \in \mathrm{BV}, k$ any integer) that, with $c_{k}^{\lambda}$ replaced by $\left|c_{k}\right|^{\lambda}$, (3.7) and (3.8) hold, respectively, for all $f \in \mathrm{BV}$ and all $f \in \mathrm{AC}$ for every $\lambda \geqslant 2$. For $0<\lambda<2$ and $f \in \mathrm{BV},(1.1)$ implies

$$
\sum_{k=-n}^{n}\left|c_{k}\right|^{\lambda} \leqslant K(\lambda) V_{f}^{\lambda / 2} n\left[\omega\left(n^{-1}, f\right)\right]^{\lambda / 2}, \quad n \geqslant 1,
$$

since the means $\left[(2 n+1)^{-1} \sum_{k=-n}^{n}\left|c_{k}\right|^{\lambda}\right]^{1 / \lambda}$ increase with $\lambda$ in $(0, \infty)$. (Here, and in the next section, $K(\cdot)$ denotes a positive constant which depends only on the displayed parameter.) Similarly, (3.6) implies that if $0<\lambda<2$ and $n \omega\left(n^{-1}, f\right) \rightarrow \infty$ $(n \rightarrow \infty)$, then

$$
\sum_{k=-n}^{n}\left|c_{k}\right|^{\lambda}=o\left(n\left[\omega\left(n^{-1}, f\right)\right]^{\lambda / 2}\right), \quad n \rightarrow \infty,
$$

for $f \in \mathrm{AC}$. In the opposite direction to (3.10) we have

TheOREM 5. Let $\psi \in \mathrm{CMT}$ and also suppose $n^{1 / 5} \psi\left(n^{-1}\right) \rightarrow \infty(n \rightarrow \infty)$. Let $(\varepsilon(n))$ be a decreasing sequence of nonnegative numbers satisfying $\varepsilon(n) \rightarrow 0(n \rightarrow \infty)$. Then, for each $\lambda \in(0,2)$, there exists $f_{\lambda} \in \mathrm{AC} \cap H[\psi]$ for which

$$
\lim _{n \rightarrow \infty} \sup \left\{\left(\sum_{k=1}^{n}\left|c_{k}\left(f_{\lambda}\right)\right|^{\lambda}\right) / \varepsilon(n) n\left[\psi\left(n^{-1}\right)\right]^{\lambda / 2}\right\}>0 .
$$

This theorem may be proved using some examples of analytic functions of class $\mathscr{P}$ constructed in [6]. A function $h$ analytic in the unit disc $U=\{z:|z|<1\}$ of the complex plane belongs to $\varphi \rho$ if $h(0)=1$ and $\operatorname{Re} h(z)>0$ for $z$ in $U$. By a result of Herglotz [4, p. 3], if $h \in \mathscr{P}$, there is a function $f$ increasing on $(-\infty, \infty)$ with $f(t+2 \pi)-f(t)=1$ for all $t$ in $(-\infty, \infty)$ such that

$$
h(z)=\int_{0}^{2 \pi} \frac{e^{i t}+z}{e^{i t}-z} d f(t)=1+4 \pi \sum_{n=1}^{\infty} c_{n}(f) z^{n}
$$

for $z$ in $U$. The function $f$ is continuous if $(1-r) A(r, h) \rightarrow 0$, where, for $0 \leqslant r<1$,

$$
A(r, h)=\sup \{\operatorname{Re} h(z):|z|=r\},
$$

and we then have, in fact,

$$
\omega(\delta, f) \leqslant \delta A(1-\delta, h), \quad 0<\delta<1,
$$

(cf. left-hand inequality of (2.6)). Using these simple facts it is not too difficult to show that the examples of functions in $\mathscr{P}$ constructed in [6] to prove Theorem 2 of that paper can be adapted to prove Theorem 5 here. We leave the details to the reader. 
4. Counterexamples for Theorem 3. In the opposite direction to (3.7) we now prove

ThEOREM 6. Let $\psi \in \mathrm{CMT}$ be such that $n \psi\left(n^{-1}\right) \rightarrow \infty(n \rightarrow \infty)$. Then there exists $f \in \mathrm{BV}^{+} \cap H[\psi]$ for which

$$
\sum_{k=1}^{n} c_{k}\left(f_{0}\right)^{\lambda} \geqslant K(\lambda) n \psi\left(n^{-1}\right) \text { for every } n, \lambda \geqslant 1 .
$$

Proof. We show that our theorem follows easily from some results of Salem $[8,9]$. Let the function $\psi$ be given as above. Let $\Phi_{2}$ be a continuous increasing function on $[0,2 \pi]$ of the type defined in [8, p. 75] and let $f_{0}(x)=\Phi_{2}(x+\pi), x \in(-\infty, \infty)$, where we assume $\Phi_{2}$ is defined for all $x$ by the condition $\Phi_{2}(x+2 \pi)-\Phi_{2}(x) \equiv K$. Then $f_{0} \in \mathrm{BV}, c_{k}\left(f_{0}\right)=(-1)^{k} c_{k}\left(\Phi_{2}\right) \geqslant 0$ for all $k$, and the argument used to prove Lemma 1 in [9] with only minor changes shows that $\Phi_{2}$ can be constructed so that $\omega\left(\delta, f_{0}\right)=\omega\left(\delta, \Phi_{2}\right)=O(\psi(\delta)), \delta \rightarrow 0^{+}$, and

$$
\sum_{k=1}^{n} c_{k}\left(f_{0}\right)^{2}=\sum_{k=1}^{n}\left|c_{k}\left(\Phi_{2}\right)\right|^{2} \geqslant K n \psi\left(n^{-1}\right)
$$

for every $n \geqslant 1$. This gives (4.1) for the case $\lambda=2$. Next suppose $q>1$ and set $p=q /(q-1)$ so that $p^{-1}+q^{-1}=1$. Then, by (4.2) and Hölder's inequality,

$$
K n \psi\left(n^{-1}\right) \leqslant \sum_{k=1}^{n} c_{k}\left(f_{0}\right)^{2} \leqslant\left(\sum_{k=1}^{n} c_{k}\left(f_{0}\right)\right)^{1 / p}\left(\sum_{k=1}^{n} c_{k}\left(f_{0}\right)^{q+1}\right)^{1 / q}
$$

so, by (3.3), we have, for $n \geqslant 1$,

$$
\sum_{k=1}^{n} c_{k}(f)^{q+1} \geqslant K(q)\left[n \psi\left(n^{-1}\right)\right]^{\left(1-p^{-1}\right) q}=K(q) n \psi\left(n^{-1}\right) .
$$

Since $c_{k}\left(f_{0}\right)=O(1), k \rightarrow \infty$, this implies (4.1) for all $\lambda \geqslant 1$ and the proof of Theorem 6 is complete.

We note that if $f \in \mathrm{BV}$ and $(\gamma(n))_{0}^{\infty}$ is an arbitrary convex sequence tending to 0 , then [11, Vol. 1, p. 179] there exists $g \in \mathrm{AC}$ such that $c_{n}(g)=\gamma(|n|) c_{n}(f)$ for every integer $n$, and we further have [9. Remark II, p. 31] that $\omega(g, \delta) \leqslant K \omega(f, \delta), \delta>0$. The next theorem, which shows that (3.8) is the best possible estimate of its kind for the class AC for every $\lambda>1$, is thus an easy consequence of Theorem 6 .

THEOREM 7. Let the function $\psi$ be defined as in Theorem 6 and let $(\varepsilon(n))$ be an arbitrary sequence of nonnegative numbers tending to 0 as $n \rightarrow \infty$. Then, for every $\lambda>1$, there exists $g_{\lambda} \in \mathrm{AC}^{+} \cap H[\psi]$ for which

$$
\sum_{k=1}^{n} c_{k}\left(g_{\lambda}\right)^{\lambda} \geqslant K(\lambda) \varepsilon(n) n \psi\left(n^{-1}\right) \text { for every } n \geqslant 1 \text {. }
$$

5. Proof of Theorem 4. The proof of the theorem depends on

Lemma 4. Let $\psi$ be as in Theorem 4. Then there is a convex sequence $\left(b_{n}\right)_{1}^{x}$ of nonnegative numbers tending to 0 such that

$$
n \psi\left(n^{-1}\right) \leqslant \sum_{m=1}^{n} b_{m} \leqslant K n \psi\left(n^{-1}\right) \text { for } n \geqslant 1 .
$$


Proof. We note first that, since $\psi \in$ CMT, (2.1) implies

$$
p \psi\left(p^{-1}\right) \leqslant 2 q \psi\left(q^{-1}\right) \text {. }
$$

if $p, q$ are positive integers with $q \geqslant p$.

We define an increasing sequence $\left(n_{k}\right)_{1}^{\infty}$ of positive integers inductively as follows. Let $n_{1}=1$ and, for $k \geqslant 1$, choose $n_{k+1}$ to be the least value of the integer $N$ for which $N \psi\left(N^{-1}\right)>2 n_{k} \psi\left(n_{k}^{-1}\right)$. Such a choice is possible as $n \psi\left(n^{-1}\right) \rightarrow \infty(n \rightarrow \infty)$. Then, for $k \geqslant 1$,

$$
n \psi\left(n^{-1}\right) \leqslant 2 n_{k} \psi\left(n_{k}^{-1}\right), \quad n_{k} \leqslant n<n_{k+1},
$$

and

$$
n_{k+1} \psi\left(n_{k+1}^{-1}\right)>2 n_{k} \psi\left(n_{k}^{-1}\right)
$$

We now set

$$
d_{1}=\psi(1), \quad d_{m}=\psi\left(n_{k+1}^{-1}\right), \quad n_{k}<m \leqslant n_{k+1}, k \geqslant 1 .
$$

Then $\left(d_{m}\right)_{1}^{\infty}$ is a decreasing sequence tending to 0 and, by (5.2)-(5.4), we have [1, Vol. 2, pp. 175-176]

$$
\sum_{m=1}^{n} \dot{d}_{m} \leqslant K n \psi\left(n^{-1}\right) \text { for } n \geqslant 1 .
$$

Also for $n_{k} \leqslant n<n_{k+1}$ and $k \geqslant 1$,

$$
\sum_{m=1}^{n} d_{m} \geqslant \sum_{m=1}^{n_{k}} d_{m} \geqslant n_{k} \psi\left(n_{k}^{-1}\right)
$$

so, by (5.3),

$$
\sum_{m=1}^{n} d_{m} \geqslant \frac{1}{2} n \psi\left(n^{-1}\right) \text { for every } n \geqslant 1 .
$$

We next write, for $m \geqslant 1$,

$$
b_{m}=2 e \sum_{k=1}^{\infty} e^{-m / k} \Delta d_{k}
$$

where $\Delta d_{k}=d_{k}-d_{k+1}$ for $k \geqslant 1$. (I am indebted to A. W. Norton for bringing this sequence to my attention some years ago.) It is easily verified that $\left(b_{m}\right)$ is a convex sequince decreasing to 0 as $n \rightarrow \infty$ and, for $m \geqslant 1$,

$$
b_{m} \geqslant 2 e \sum_{k=m}^{\infty} e^{-m / k} \Delta d_{k} \geqslant 2 \sum_{k=m}^{\infty} \Delta d_{k},
$$

that is,

$$
b_{m} \geqslant 2 d_{m} \text { for } m \geqslant 1 \text {. }
$$

Furthermore,

$$
b_{m} \leqslant 2 e \sum_{k=1}^{m} e^{-m / k} \Delta d_{k}+2 e d_{m}
$$


and, since, for $n \geqslant 1$,

$$
\begin{aligned}
\sum_{m=1}^{n} \sum_{k=1}^{m} e^{-m / k} \Delta d_{k} & =\sum_{k=1}^{n} \Delta d_{k} \sum_{m=k}^{n} e^{-m / k} \leqslant e^{-1} \sum_{k=1}^{n} \Delta d_{k}\left(1-e^{-1 / k}\right)^{-1} \\
& \leqslant 2 e^{-1} \sum_{k=1}^{n} k \Delta d_{k} \leqslant 2 e^{-1} \sum_{k=1}^{n} d_{k},
\end{aligned}
$$

it follows that

$$
\sum_{m=1}^{n} b_{m} \leqslant K \sum_{m=1}^{n} d_{m} \text { for } n \geqslant 1
$$

Clearly, (5.1) now follows from (5.8), (5.7), (5.6) and (5.5), and the proof of Lemma 4 is complete.

Now let $\left(b_{n}\right)_{1}^{\infty}$ be the sequence obtained above and set $b_{0}=2 b_{1}$. Then $\left(b_{n}\right)_{0}^{\infty}$ is a convex sequence tending to 0 and, hence [11, Vol. 1, p. 183], there is a nonnegative $2 \pi$-periodic function $h \in L(0,2 \pi)$ such that $\frac{1}{2} b_{0}+\sum_{1}^{\infty} b_{n} \cos n x$ is the Fourier series of $h$. If we write

$$
f(x)=\int_{0}^{x} h(t) d t_{\infty} \quad x \in(-\infty, \infty)
$$

then the increasing function $f$ belongs to $\mathrm{AC}, c_{n}(f)=b_{n}$ and $c_{-n}=c_{n} \quad(n=$ $0,1,2, \ldots)$, and, by the left-hand inequality of (2.6),

$$
K_{1} n \omega\left(n^{-1}, f\right) \leqslant \sup \left\{\sigma_{n}(x, f): 0 \leqslant x \leqslant 2 \pi\right\} \leqslant 2 \sum_{k=0}^{n} b_{k} \leqslant 6 \sum_{k=1}^{n} b_{k},
$$

since $b_{0}=2 b_{1}$. It now follows from the right-hand inequality of $(5.1)$ that $\omega\left(n^{-1}, f\right)$ $\leqslant K \psi\left(n^{-1}\right), n \geqslant 1$, from which we readily deduce that $f \in H[\psi]$. Since the left-hand inequality of (5.1) gives (3.9), the proof of Theorem 4 is complete.

We finish by showing, as promised in $\S 3$, there is a periodic function $g \in \mathrm{BV}$ for which (3.5) is divergent while (3.4) is convergent. To do this we define a sequence $\left(\alpha_{n}\right)_{0}^{\infty}$ as follows:

$$
\alpha_{0}=3, \quad \alpha_{n}=[\log (n+1)]^{-2}, \quad n \geqslant 1 .
$$

Then $\left(\alpha_{n}\right)$ is a convex sequence tending to 0 and so, as we have noted earlier, there is an increasing function $g \in \mathrm{AC}$ for which $c_{n}(g)=\alpha_{n}$ and $c_{-n}(g)=c_{n}(g), n=$ $0,1,2, \ldots$ Also, by the right-hand inequality of (2.6) and the monotonicity of $\omega$,

$$
\begin{aligned}
K n \omega\left(n^{-1}, g\right) & \geqslant \sigma_{2 n}(0)=\sum_{k=-2 n}^{2 n}\left(1-\frac{|k|}{2 n}\right) c_{k}(g) \\
& \geqslant \frac{1}{2} \sum_{-n}^{n} c_{k}(g) \geqslant \sum_{1}^{n} \alpha_{k} \geqslant K_{1} n[\log (n+1)]^{-2}
\end{aligned}
$$

by an elementary estimation. Hence

$$
\omega\left(n^{-1}, g\right) \geqslant K[\log (n+1)]^{-2}, \quad n \geqslant 1,
$$


and (3.5) diverges as required. Since $c_{n}\left(g^{*}\right)=\left|c_{n}(g)\right|^{2}, n=0, \pm 1, \pm 2, \ldots$, it follows easily by arguments similar to those used above involving the left-hand inequality of (2.6) that

$$
\omega\left(n^{-1}, g^{*}\right) \leqslant K[\log (n+1)]^{-4}, \quad n \geqslant 1 .
$$

(3.4) therefore converges and we are finished.

\section{REFERENCES}

I. N. K. Bari, Trigonometric series, Vols. 1, 2, Fizmatgiz, Moscow, 1961; English transl., Macmillan, New York and Pergamon, Oxford, 1964.

2. R. P. Boas, Fourier series with positive coefficients, J. Math. Anal. Appl. 17 (1967), 463-483.

3. S. V. Bockarev, On a problem of Zigmund, Izv. Akad. Nauk SSSR Ser. Math. 37 (1973), 630-638: English transl., Math. USSR-Izv. 7 (1973).

4. P. L. Duren, Theory of $H^{p}$ spaces, Academic Press, New York, 1970)

5. (j. H. Hardy and W. W. Rogosinski, Fourier series, 3rd ed., Cambridge Univ. Press, Cambridgc, 1965.

6. F. Holland and J. B. Twomey, On coefficient means of certain subclasses of univalent functions, Trans. Amer. Math. Soc. 185 (1973), 151-163.

7. Integral means of functions with positive real part, Canad. J. Math. 32 (1980), 1008-1020.

8. R. Salem, On singular monotonic functions of the Cantor type, Math. Phys. 21 (1942), 69-82.

9. On a theorem of Zygmund, Duke Math. J. 10 (1943), 23-31.

10. I. Wik, Criteria for absolute convergence of Fourier series of functions of hounded variation. Trans. Amer. Math. Soc. 163 (1971), 1-24.

11. A. Zygmund, Trigonometric series, Vols. 1, 2, 2nd rev. ed., Cambridge Univ. Press, New York, 1959.

Department of Mathematics, University College, Cork, Ireland 